\title{
Frühjahrstagung der Arbeitsgemeinschaft Knochentumoren 2019
}

\section{Tagungsbericht}

Die 93. Tagung der AG Knochentumoren fand in Zagreb/Kroatien auf Einladung von Prof. Sven Seiwerth vom 24.bis 25. Mai in den Räumen des Pathologischen Instituts der Medizinischen Fakultät statt. Zusammen mit seinem Team hatte Prof. Seiwerth die Tagung exzellent vorbereitet und organisiert und ein abwechslungsreiches Programm zusammengestellt, so dass es allen Gästen möglich war, Zagreb und seine Umgebung besser kennenzulernen.

Die Tagung begann am 24.5. mit der Vorstandssitzung (15.00 bis 16.00 Uhr) gefolgt von der Präsentation von ad hoc-Fällen, die aktuelle diagnostische oder therapeutische Probleme beinhalteten und entweder vorab angemeldet (sekretariat@agkt.org) oder spontan mitgebracht wurden. Insgesamt wurden 5 Fälle vorgestellt. Zunächst wurde der Fall einer 37-jährigen Patientin diskutiert, die seit 4-5 Wochen über paravertebrale Rippenschmerzen klagte. In der Bildgebung fand sich eine multilokuläre Osteolyse ohne sichere begleitende Mineralisation. Unter der radiologischen Verdachtsdiagnose einer möglicherweise sekundär veränderten fibrösen Dysplasie oder einer fokalen hämatopoetischen Hyperplasie der Rippe wurde zunächst zu einer Biopsie geraten (nach Tagungsende wurde histologisch eine fibröse Dysplasie gesichert).

Ein weiterer Fall betraf einen 64-jährigen Patienten, der sich mit einer Schwellung am rechten Unterschenkel vorstellte. Vor 20 Jahren erlitt der Patient einen Unfall mit Tibiafraktur rechts. Intraoperativ fand sich krümeliges Material, die Mikrobiologie war negativ. In der Diskussion wurde ein Tumor als extrem unwahrscheinlich angesehen und die vorliegende Bildgebung zusammen mit den klinischen Befunden einer alten Kompartmentnekrose zugeordnet.
Als dritter Fall wurde ein 25-jähriger Patient vorgestellt, der körperlich und geistig beeinträchtigt war und bei dem nach Fremdanamnese seit 7 Jahren eine langsam zunehmende Schwellung des rechten Unterschenkels bestand. Radiologisch fanden sich blasig-osteolytische Auftreibungen der distalen rechten Tibia (Markraum und Periost) sowie der (leider nur teilweise erfassten) Fußwurzelknochen und des distalen Femurs (partieller Nachweis in einzelnen MR-Sequenzen). In der Biopsie (Tibia) zeigte sich ein Knorpeltumor ohne invasiv-destruktives Wachstum. Um einen M. Ollier auszuschließen, wurde eine erweiterte Bildgebung (GanzkörperMRT, komplette Darstellung des Fußes und des distalen Femurs) empfohlen.

Der vierte Fall betraf eine 74-jährige Patientin mit Schmerzen in der rechten Schulter. Im CT fand sich eine - szintigraphisch aktive - Osteolyse im proximalen Humerus, welche radiologisch zunächst als Metastase oder Myelommanifestation gedeutet wurde. Außerdem bestanden Bursitis-artige Veränderungen am Schultergelenk, welche in erster Linie für die Schmerzsymptomatik als ursächlich angesehen wurden. Die Biopsie der Humerusläsion (marginale Ausräumung) ergab eine Infiltration durch schaumzellige Histiozyten (pos: CD 68, CD 163; neg: CD1a, S-100, Langerin) sowie eine begleitende Fibrose. Eine atypische Mykobakteriose oder eine Erdheim Chester-Erkrankung sollten trotz ungewöhnlicher Manifestation noch ausgeschlossen (BRAF p.600VE-Mutation) und der Fall an der nächsten Sitzung in München diskutiert werden.

Als 5. Fall wurden die Befunde eines 50-jährigen Patienten diskutiert, der sich nach einem Sturz eine Schenkelhalsfraktur zuzog, welche sich in der Bildgebung als pathologische Fraktur erwies. Die Bildgebung wurde zunächst als dedifferenziertes Chondrosarkom gedeutet. Die Biopsie ergab ein Klarzellchondrosarkom ohne histologische Hinwei- se auf eine Dedifferenzierung, eine Nachresektion ergab einen histologisch identischen Resttumor, so dass wegen der Diskrepanz in der Aggressivitätsbeurteilung zwischen Radiologie und Pathologie (high-grade Tumor vs. low-grade Tumor) zunächst engmaschige Kontrollen empfohlen wurden.

In einer anschließenden Kurzpräsentation stellte Prof. Jundt noch die Basler Erfahrungen mit Antikörpern gegen Caldesmon vor. Dieses Molekül wird als Marker für glatte Muskulatur angesehen. Es existieren jedoch unterschiedliche Caldesmon-Formen. Nur das hochmolekulare Caldesmon ist Glattmuskel-spezifisch, niedermolekulares Caldesmon kommt auch in anderen Zellen (z. B. Myofibroblasten) vor [Dabrowska R et al. Protoplasma (2004) 224: 1-13]. Nach den Basler Erfahrungen und den Angaben in der Literatur (Beck, EM et al. J Cutan Pathol. 2018;45:581-587) eignet sich der Clone h-CD (Dako) am besten für die Markierung glatter Muskulatur.

Nach Diskussion der ad hoc-Fälle fanden die wissenschaftlichen Vorträge statt, welche einen Überblick über 20 Jahre orthopädische Pathologie an der Universität Zagreb, die Epidemiologie muskuloskeletaler Tumoren sowie die Problematik der Behandlung und Diagnostik von Knochentumoren in Kroatien beinhalteten:

- Orthopedic pathology - 20 years single center experience:

Sven Seiwerth (Institute of Pathology University of Zagreb School of Medicine and KBC Zagreb- Die Kurzfassung des Vortrags ist im Anschluss an den Tagungsbericht auf Seite 229 zu finden).

- Pediatric bone tumors in Croatia, a single oncology centre experience: Bonevski $A^{1}$, Stepan Giljević ] ${ }^{1}$, Ćepulić $\mathrm{M}^{1}$, Seiwerth $\mathrm{S}^{2}\left({ }^{1}\right.$ Children's Hospital Zagreb, Oncology department, Zagreb, Croatia $^{2}$, Institute of Pathology School of 
Medicine University of Zagreb, Zagreb, Croatia - Die Kurzfassung des Vortrags ist im Anschluss an den Tagungsbericht auf Seite 231 zu finden)

- Epidemiology of musculoskeletal tumors in a national referral orthopedic department. A study of 3482 cases: Bergovec $\mathrm{M}^{1,2}$, Kubat $\mathrm{O}^{1}$, Smerdelj $\mathrm{M}^{1}$, Seiwerth $\mathrm{S}^{3}$, Bonevski $\mathrm{A}^{4}$, Orlic $\mathrm{D}^{1}$ ('Department of Orthopaedic Surgery, University of Zagreb School of Medicine, KBC Zagreb, ${ }^{2}$ Department of Orthopedic Surgery, Medical University of Graz, ${ }^{3}$ Clinical Department of Pathology and Cytology, Medical School University of Zagreb, KBC Zagreb, ${ }^{4}$ Children's University Hospital Zagreb - aktualisierte Fassung der in Cancer Epidemiology 2015; 39: 298-302 bereits publizierten $\mathrm{Ar}$ beit - PMID:25703268)

Das anschließende gemeinsame Abendessen fand in den Räumen des 1927 auf Initiative von Andrija Štampar mit Unterstützung der Rockefeller Foundation errichteten «Institutes for Hygiene and School of Public Health" statt, das 2014 nach seinem Gründer in "Andrija Štampar School of Public Health» umbenannt wurde. Štampar war außerdem 1948 einer der Initiatoren der WHO und Präsident der ersten Weltgesundheitsversammlung. Die jetzige Direktorin, Frau Professor Mirjana Kujundžić Tiljak, stellte in einem kurzen Abriss die Institutsgeschichte dar und gab uns außerdem die Gelegenheit, das Institut zu besichtigen.

\section{Mitgliederversammlung}

Am Samstagvormittag begann die nichtöffentliche Mitgliederversammlung um $8.30 \mathrm{Uhr}$ (bis $9.30 \mathrm{Uhr}$ ).

Vor Eintritt in die Tagesordnung gedachten die Teilnehmer ihres im März verstorbenen Mitgliedes Prof. Dr. Peter Meister aus Mün- chen, der seit 1976 Mitglied der AG Knochentumoren war, die Sitzungen unserer Gesellschaft mit seinen Beiträgen geprägt und in seiner speziellen Art oft belebt hat.

Die Homepage der AGKT wurde überarbeitet und den neuen Datenschutzerfordernissen angepasst. Außerdem wurde ein Abschnitt zur Geschichte der AGKT eingefügt.

Als neue Mitglieder wurden Herr Dr. Manoj Kakkassery, Leiter Muskuloskelettale Radiologie am Helios-Klinikum Berlin-Buch und Frau PD Dr. Sylvia Hoeller, Oberärztin am Institut für Pathologie des Universitätsspitals Basel, in die AGKT aufgenommen.

\section{Wissenschaftliche}

\section{Falldiskussion}

Hauptprogrammpunkt der samstäglichen Sitzung war die wissenschaftliche Falldiskussion. Insgesamt wurden 9 neue Fälle diskutiert sowie Nachträge (Verläufe) zu 5 bereits vorgestellten Fällen präsentiert. 6 Fälle betrafen die langen Röhrenknochen ( $3 \times$ Tibia, $2 \times$ Femur, $1 \times$ Ulna), je ein Fall die Hände bzw. den Fuß/Unterschenkel und den Schädel. Siebenmal waren Männer betroffen, zweimal Frauen. Die Patienten waren bei Symptombeginn bzw. Diagnose (1 Zufallsbefund) zwischen 11 und 66 Jahre alt.

5 Tumoren waren gutartig (darunter ein nicht sicher klassifizierbarer Gefäßtumor/ Spindelzellhämangiom, eine aneurysmatische Knochenzyste, ein Chondromyxoidfibrom, ein sogenannter Kalottendefekt bei Neurofibromatose 1 und gleichzeitig bestehender fibröser Dysplasie, und ein brauner Tumor bei Hyperparathyreoidismus). Der letztgenannte Tumor wurde nur zufällige anlässlich einer Traumaabklärung entdeckt. In 4 Fällen lagen bösartige Tumoren vor (ein extraaxiales, intraartikuläres Chordom, ein fibroblastisches high-grade Osteo- sarkom, ein Grad2-Chondrosarkom und ein im Knochen extrem selten vorkommender sogenannter inflammatorischer myofibroblastischer Tumor/IMT). Die histologisch gestellte Verdachtsdiagnose: IMT wurde molekularbiologisch durch das typische Methylierungsprofil untermauert (Tomassen T, et al.: Ann Diagn Pathol. 2019;41:102-105).

Am Ende der Veranstaltung erhielten Frau Dr. Boxberg, Frau PD Dr. Specht, Frau Dr. C. Knebel, Prof. Wörtler (alle TU München) und Prof. Dr. Barth (Uniklinik Ulm) den Preis für die beste Fallpräsentation (Fall 1106: extraaxiales intraartikuläres Chordom - bereits publiziert in Skeletal Radiology - PMID 31104146).

Der offizielle Teil wurde gegen 13.30 Uhr mit einem herzlichen Dank für die hervorragend organisierte und sehr persönlich gestaltete Tagung an die Veranstalter beendet, deren Einsatz vielen Teilnehmenden Zagreb und Kroatien sicher sehr viel näher gebracht haben dürfte. Mit einem nachmittäglichen Ausflug ins Neandertaler Museum in Krapina, einem anschließenden gemeinsamen Abendessen sowie mit einer geführten Tour durch Zagreb am Sonntagmorgen ging eine sehr interessante, zu Diskussionen anregende und äußerst abwechslungsreiche Veranstaltung zu Ende.

\section{Zukünftige Tagungen}

Die Herbsttagung 2019 findet am 18./19.10. in München statt. Veranstaltungsort der Frühjahrstagung 2020 (27./28.3.) ist Ulm.

Falls Interesse besteht, als Gast an den Tagungen der AG Knochentumoren teilzunehmen, können weitere Informationen über das Sekretariat der AGKT in Basel oder über die Homepage abgerufen werden. 